\title{
Preference profiles determining the proposals in the Gale-Shapley algorithm for stable matching problems
}

\author{
Noriyoshi Sukegawa - Yoshitsugu Yamamoto
}

Received: date / Accepted: date

\begin{abstract}
Concerning the strategic manipulability of the the stable matching produced by the Gale-Shapley algorithm, Kobayashi and Matsui recently considered the existence problem of a preference profile of women, that is, given a preference profile of men, find a preference profile of women that makes the Gale-Shapley algorithm produce the prescribed complete matching of men and women. Reformulating this problem by introducing the set of proposals to be made through the execution of the algorithm, and switching the roles of men and women, we consider the existence problem of a preference profile of men and show that the problem is reduced to a problem of checking if a directed graph is a rooted tree and it is solvable in polynomial time. We also show that the existence problem of preference profiles of both sexes when a set of proposals is given is solvable in polynomial time.
\end{abstract}

Keywords Stable matching · Gale-Shapley algorithm · Preference profile $\cdot$ Strategic manipulability $\cdot$ Rooted spanning tree $\cdot$ Matroid intersection

\section{Introduction}

Those who triggered this work are Tomomi Matsui, who presented his work concerning the strategic issue in the stable matching model, and Akihisa Tamura, who raised an incisive question for his presentation. Matsui's work has been published in [7], where given a preference profile of men and a complete matching, they consider the

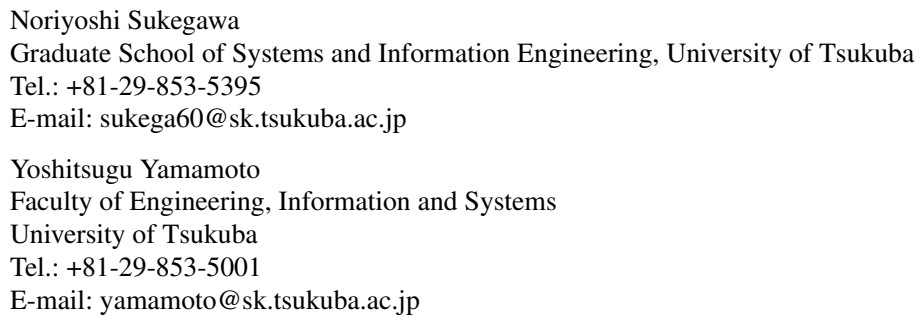


problem of finding a preference profile of women such that the men-proposing GaleShapley algorithm produces the given complete matching, and show that the problem is solvable in polynomial time. They also consider several variations of the problem in [8], and show that one of them results in NP-completeness.

Suppose that given a preference profile of women and a complete matching, we are asked if there is a preference profile of men such that the men-proposing GaleShapley algorithm produces the given complete matching. This problem has a trivial solution, that is, each man just has to rank his assigned mate first in his preference list. When we are given a preference profile of men, being given a complete matching is equivalent to being given the set of women to whom each man proposes to during the execution of the algorithm. Hence the problem that Kobayashi and Matsui consider in [7], which is the first problem in [8], can be restated as "given a preference profile of men and a set of proposals, find a preference profile of women such that during the execution of the men-proposing Gale-Shapley algorithm each man proposes to women prescribed by the set of proposals." The aim of this paper is to answer the question what if we switch the roles of men and women in this setting. We will show that it reduces to a problem of determining if a directed graph is a rooted spanning tree, hence is solvable in polynomial time.

The issue of strategical manipulability in the stable matching model has been discussed in many publications such as [5], [10], and the references therein. To our knowledge, the above problem setting is novel and will serve as a foundation stone for further research on the strategical manipulability in the stable matching model. In the next section, we describe the stable matching problem and the men-proposing GaleShapley algorithm. In Section 3 we define the problem considered in this paper, and then in Section 4 introduce the keystone of this paper named second suitor graph. The solution as well as the polynomial solvability of the problem is shown in Section 5. In Section 6 we discuss the existence of preference profiles of men and women when a set of proposals is prescribed.

\section{Stable matching and men-proposing Gale-Shapley algorithm}

We denote the set of men and the set of women by $M$ and $W$, respectively, and suppose that they consist of the same number, say $n$, of persons, i.e., $|M|=|W|=n$. Each man has a totally ordered list of all the women, which we call his preference list, and each woman also has her preference list of all the men. We denote person $u$ 's preference list by $L_{u}$. We denote $v \succ_{u} v^{\prime}$ or $v^{\prime} \prec_{u} v$ when $v$ is ranked prior to $v^{\prime}$ in $L_{u}$, and $v \succeq_{u} v^{\prime}$ or $v^{\prime} \preceq_{u} v$ when $v=v^{\prime}$ or $v \succ_{u} v^{\prime}$. We will use the symbols $L_{u}$ and $\succ_{u}$ interchangeably. We also denote the set of preference lists of $M$ and $W$ by $\mathscr{L}_{M}:=\left[L_{m}\right]_{m \in M}=\left[\succ_{m}\right]_{m \in M}$ and $\mathscr{L}_{W}:=\left[L_{w}\right]_{w \in W}=\left[\succ_{w}\right]_{w \in W}$, and call them preference profile of men and preference profile of women, respectively.

Definition 1 A complete matching is a mapping $f: M \cup W \rightarrow M \cup W$ such that

1. $f(m) \in W$ for all $m \in M$, and $f(w) \in M$ for all $w \in W$,

2. $w=f(m)$ if and only if $m=f(w)$ for all $(m, w) \in M \times W$. 
We say that $f(u)$ is $u$ 's mate in $f$ and $\{u, f(u)\}$ is a matched pair in $f$. We say $\{m, w\}$ with $m \in M$ and $w \in W$ is an unmatched pair in $f$ when $w \neq f(m)$.

Definition 2 An unmatched pair $\{m, w\}$ in a complete matching $f$ is said to be a blocking pair for $f$ if and only if

$$
w \succ_{m} f(m) \text { and } m \succ_{w} f(w) .
$$

Definition 3 A complete matching $f$ is said to be a stable matching if and only if it admits no blocking pairs.

Gale and Shapley [4] showed that there is a stable matching for any given pair of preference profiles $\mathscr{L}_{M}$ and $\mathscr{L}_{W}$. Their constructive proof is based on an algorithm now known as the Gale-Shapley algorithm which repeats a proposal followed by an engagement or a decline. The algorithm has two variations: men-proposing and women-proposing depending on which sex proposes to the other sex. The version we consider in this paper is the men-proposing Gale-Shapley algorithm (mGS for short) described below, where $k$ is the iteration counter which will be used in the proof of Lemma 5.

\section{Men-proposing Gale-Shapley algorithm (mGS)}

Step 0: Set $F_{M}:=M, F_{W}:=W, \Pi=\emptyset, \mu(u)=u$ for all $u \in M \cup W$, and $k:=0$.

Step 1: If $F_{M}=\emptyset$, then output $\mu$ and $\Pi$, and stop.

Step 2: Choose $m \in F_{M}$, let

$$
\begin{aligned}
& w:=\max _{\succ_{m}}\left\{w^{\prime} \in W \mid\left(m, w^{\prime}\right) \notin \Pi\right\}, \\
& \Pi:=\Pi \cup\{(m, w)\} .
\end{aligned}
$$

Step 3:

3a: If $w \in F_{W}$, then set

$$
\begin{aligned}
& \mu(m):=w \quad \text { and } \quad \mu(w):=m, \\
& F_{M}:=F_{M} \backslash\{m\} \quad \text { and } \quad F_{W}:=F_{W} \backslash\{w\},
\end{aligned}
$$

and go to Step 4.

3b: If $w \notin F_{W}$, let $m^{\prime}:=\mu(w)$.

3b1: If $m \succ_{w} m^{\prime}$, then set

$$
\begin{aligned}
& \mu(m):=w \text { and } \quad \mu(w):=m, \\
& \mu\left(m^{\prime}\right):=m^{\prime}, \\
& F_{M}:=F_{M} \backslash\{m\} \cup\left\{m^{\prime}\right\}
\end{aligned}
$$

and go to Step 4.

3b2: If $m \prec_{w} m^{\prime}$, go to Step 4.

Step 4: Update $k:=k+1$ and return to Step 1 . 
In the execution of the algorithm each person is either engaged or free. In the above description $F_{M}$ and $F_{W}$ are the sets of men and women who are free at the current iteration, respectively, and $\Pi$ is the set of ordered pairs of man $m$ and woman $w$ such that $m$ has proposed to $w$ up to the current iteration. A man $m$ who is free is chosen in Step 2, and then let him propose to his most favorite woman $w$ whom he has not yet proposed to. If the woman $w$ prefers the proposer $m$ to her current mate $m^{\prime}$, she breaks her current engagement, sets her ex-mate $m^{\prime}$ free and becomes engaged to $m$ in Step 3 .

Definition 4 For a given pair of preference profiles $\mathscr{L}_{M}$ and $\mathscr{L}_{W}$, we denote the matching $\mu$ and the set of proposals $\Pi$ that the mGS produces by $\mu\left(\mathscr{L}_{M}, \mathscr{L}_{W}\right)$ and $\Pi\left(\mathscr{L}_{M}, \mathscr{L}_{W}\right)$, respectively.

It is known that the matching $\mu\left(\mathscr{L}_{M}, \mathscr{L}_{W}\right)$ as well as the set of proposals $\Pi\left(\mathscr{L}_{M}, \mathscr{L}_{W}\right)$ is independent of the choice of a man $m \in F_{M}$ in Step 2. See Theorem 1.2.2 in Gusfield and Irving [5] or Lecture 2 in Knuth [6].

Definition 5 For a nonempty subset $P$ of $M \times W$ let

$$
\begin{array}{ll}
P_{M}(m):=\{w \in W \mid(m, w) \in P\} & \text { for } m \in M, \\
P_{W}(w):=\{m \in M \mid(m, w) \in P\} & \text { for } w \in W .
\end{array}
$$

We call $P \subseteq M \times W$ a set of proposals when $P_{M}(m)$ and $P_{W}(w)$ are nonempty for all $m \in M$ and $w \in W$.

Since each man proposes to women who are successively less preferred by him, and each woman who receives a proposal compares her current mate with the proposer and becomes engaged to a more favorite man, we readily obtain the following lemma.

Lemma 1 For $\mathscr{L}_{M}:=\left[\succ_{m}\right]_{m \in M}$ and $\mathscr{L}_{W}:=\left[\succ_{w}\right]_{w \in W}$, let $\mu:=\mu\left(\mathscr{L}_{M}, \mathscr{L}_{W}\right)$ and $\Pi:=\Pi\left(\mathscr{L}_{M}, \mathscr{L}_{W}\right)$. Then

$$
\begin{aligned}
& \mu(m)=\min _{\succ_{m}} \Pi_{M}(m) \quad \text { for } m \in M, \\
& \mu(w)=\max _{\succ_{w}} \Pi_{W}(w) \quad \text { for } w \in W,
\end{aligned}
$$

where $\min _{\succ_{m}} \Pi_{M}(m)$ is the woman who is ranked lowest in the set $\Pi_{M}(m)$ according to man m's preference $\succ_{m}$, and $\max _{\succ_{w}} \Pi_{W}(w)$ is the man who is ranked highest in the set $\Pi_{W}(w)$ according to woman $w$ 's preference $\succ_{w}$.

\section{Problem description}

We consider the following problem in this paper.

Input : $\quad$ A preference profile of women $\mathscr{L}_{W}:=\left[\succ_{w}\right]_{w \in W}$ and a set of proposals $P \subseteq M \times W$.

Output: If there is a preference profile of men $\mathscr{L}_{M}$ such that $\Pi\left(\mathscr{L}_{M}, \mathscr{L}_{W}\right)=P$, then output $\mathscr{L}_{M}$. Otherwise, return "none exists." 
Now for given $\mathscr{L}_{W}:=\left[\succ_{w}\right]_{w \in W}$ and $P \subseteq M \times W$, let

$$
\alpha(w):=\max _{\succ_{w}} P_{W}(w)
$$

for $w \in W$. If $\alpha(w)=\alpha\left(w^{\prime}\right)$ for different women $w$ and $w^{\prime}$, the mGS would not produce $P$ no matter what preference profile of men is given. Henceforth we assume that $\alpha: W \rightarrow M$ is an injection, i.e.,

$$
\alpha(w) \neq \alpha\left(w^{\prime}\right) \text { whenever } w \neq w^{\prime} .
$$

Since $M$ and $W$ are of the same cardinality, the woman $w$ such that $\alpha(w)=m$ is uniquely determined for each $m \in M$, hence we denote it by $\alpha^{-1}(m)$. If the mGS produces $P$ for some $\mathscr{L}_{M}:=\left[\succ_{m}\right]_{m \in M}$, it satisfies the conditions

$$
\alpha^{-1}(m)=\min _{\succ_{m}} P_{M}(m)
$$

and

$$
\alpha^{-1}(m) \succ_{m} w^{\prime} \text { for all } w^{\prime} \in W \backslash P_{M}(m) .
$$

Namely, the preference list of $m$ should be as shown in Table 1 .

Table 1 Man $m$ 's preference satisfying (3) and (4)

$$
P_{M}(m) \backslash\left\{\alpha^{-1}(m)\right\} \quad \succ_{m} \quad \alpha^{-1}(m) \quad \succ_{m} \quad W \backslash P_{M}(m)
$$

A natural question would be whether such a preference profile of men $\mathscr{L}_{M}$ always gives the prescribed set of proposal $P$. In other words, "are the conditions (2), (3) and (4) on $\mathscr{L}_{M}$ sufficient for the mGS to produce $P$ ? If not, what other conditions are needed?" The following small example shows that the conditions (2), (3) and (4) are not sufficient.

Example 1 Let $M:=\{1,2\}, W:=\{a, b\}, \mathscr{L}_{M}$ and $\mathscr{L}_{W}$ be given in Table 2, and $P:=\{(1, a),(1, b),(2, a),(2, b)\}$. The underlined elements denote the prescribed proposals $P$ and the boldfaced figure in each row of $\mathscr{L}_{W}$ is $\alpha(w)$, and the boldfaced alphabet in each row of $\mathscr{L}_{M}$ is $\alpha^{-1}(m)$. Note that this instance satisfies the conditions (1), (2), (3) and (4). The mGS, however, will produce $\Pi\left(\mathscr{L}_{M}, \mathscr{L}_{W}\right)=\{(1, b),(2, a)\}$, which is different from the prescribed proposals $P$.

Table $2 \mathscr{L}_{M}$ and $\mathscr{L}_{W}$

\begin{tabular}{c|ccc|cc}
$m$ & \multicolumn{2}{|c|}{$\mathscr{L}_{M}$} & & $w$ & \multicolumn{2}{|c}{$\mathscr{L}_{M}$} \\
\hline 1 & $\underline{b}$ & $\underline{\mathbf{a}}$
\end{tabular}$\quad$\begin{tabular}{c|cc}
$a$ & $\underline{\mathbf{1}}$ & $\underline{2}$ \\
2 & $\underline{a}$ & $\underline{\mathbf{b}}$
\end{tabular}$\quad \begin{array}{lll}b & \underline{\mathbf{1}}\end{array}$


4 Second suitor graph

Definition 6 For woman $w \in W$ with $\left|P_{W}(w)\right| \geq 2$ let

$$
\beta(w):=\max _{\succ_{w}}\left(P_{W}(w) \backslash\{\alpha(w)\}\right) .
$$

Definition 7 Let $G\left(\mathscr{L}_{W}, P\right)$ be a directed bipartite graph with node set $(M \cup\{r\}) \cup W$ and arc set consisting of the following three disjoint arc sets:

$$
\begin{aligned}
& A:=\left\{(w, \alpha(w)) \in W \times M|| P_{W}(w) \mid \geq 1\right\}, \\
& B:=\left\{(\beta(w), w) \in M \times W|| P_{W}(w) \mid \geq 2\right\}, \\
& R:=\left\{(r, w) \in\{r\} \times W|| P_{W}(w) \mid=1\right\} .
\end{aligned}
$$

We call $G\left(\mathscr{L}_{W}, P\right)$ the second suitor graph for $\mathscr{L}_{W}$ and $P$.

Definition 8 For an arc $(u, v)$ of a directed graph, we call $u$ and $v$ endpoints of the arc, and we say that the arc emanates from node $u$ and terminates at node $v$. The arc $(u, v)$ is an outgoing arc of node $u$ and an incoming arc of node $v$. The indegree of node $u$, denoted by indeg $(u)$, is the number of incoming arcs of node $u$, and its outdegree, denoted by outdeg $(u)$, is the number of outgoing arcs.

Lemma 2 Suppose that (2) holds. Then the second suitor graph $G\left(\mathscr{L}_{W}, P\right)$ has the following properties.

1. $\operatorname{indeg}(r)=0$,

2. $\operatorname{indeg}(w)=\operatorname{outdeg}(w)=1$ for all $w \in W$, and

3. $\operatorname{indeg}(m)=1$ for all $m \in M$.

Proof Each node $w \in W$ has only one outgoing arc $(w, \alpha(w))$, and only one incoming arc which is either $(\beta(w), w)$ or $(r, w)$ depending on the cardinality of $P_{W}(w)$. Thus both of $\operatorname{indeg}(w)$ and $\operatorname{outdeg}(w)$ are one. The indegree of node $m \in M$ is clearly one from (2).

Definition 9 A sequence of $\operatorname{arcs} a_{1}, a_{2}, \ldots, a_{\ell}$ of a directed graph is said to be a path when $\ell \geq 2$, and $a_{i}$ has one endpoint in common with $a_{i-1}$ and its other endpoint in common with $a_{i+1}$ for $i=2,3, \ldots, \ell-1$. A path is said to be a cycle when the two end nodes of the path are the same node. When arc $a_{i+1}$ emanates from the node that $a_{i}$ terminates at for $i=1,2, \ldots, \ell-1$, we call them a directed path and a directed cycle, respectively.

Example 2 The second suitor graph $G\left(\mathscr{L}_{W}, P\right)$ for the preference profiles of Example 1 consists of two components: one being the root $r$ alone, and the other being a directed cycle passing the nodes $a, 1, b, 2$ and $a$ in this order.

Definition 10 A node $v$ of a directed graph is called a root if all the nodes are reached by directed paths starting from $v$. A rooted spanning tree is defined as a spanning tree that has a root. For each node $u$ of a rooted spanning tree, there is a unique directed path from the root to $u$. The number of arcs on this directed path is called the height of node $u$ and denoted by $h(u)$. The node right prior to $u$ on this directed path is called a predecessor of $u$ and denoted by $\operatorname{pred}(u)$, and the node right after $u$ on this directed path is called a successor of $u$ and denoted by $\operatorname{succ}(u)$. 
The following lemma is among the equivalent characterizations of rooted spanning tree ${ }^{1}$ given in Berge [1].

Lemma 3 (Theorem 13 in Chapter 3, Berge [1]) A directed graph is a rooted spanning tree with root $v$ if and only if

1. $\operatorname{indeg}(v)=0$,

2. indeg $(u)=1$ for all nodes $u \neq v$, and

3. the graph contains no cycles.

Lemma 4 The second suitor graph $G\left(\mathscr{L}_{W}, P\right)$ is a rooted spanning tree with root $r$ if and only if it contains no directed cycles.

Proof Since the "only if" part is trivial, we prove the "if" part. Suppose $G\left(\mathscr{L}_{W}, P\right)$ is not a rooted spanning tree. Then by Lemma 2 and Lemma 3 it contains a cycle, say $C$. If $C$ contains the root $r$, whose indegree is zero, $C$ has a node whose indegree is more than one. This contradicts Lemma 2. Then the node set of $C$ is contained in $M \cup W$. Since the indegrees are one for all the nodes of $M \cup W$ by Lemma 2, this implies that the cycle $C$ is a directed cycle.

\section{Existence of men's preference profile}

Lemma 5 Suppose that for a given set of proposals $P \subseteq M \times W$ there is a preference profile of men $\mathscr{L}_{M}$ such that $P=\Pi\left(\mathscr{L}_{M}, \mathscr{L}_{W}\right)$. Then the second suitor graph $G\left(\mathscr{L}_{W}, P\right)$ is a rooted spanning tree.

Proof We will show that the existence of a directed cycle in the second suitor graph $G\left(\mathscr{L}_{W}, P\right)$ would lead to a contradiction. Denote a directed cycle by

$$
\left(w_{1}, m_{1}\right),\left(m_{1}, w_{2}\right), \ldots,\left(m_{i-1}, w_{i}\right),\left(w_{i}, m_{i}\right),\left(m_{i}, w_{i+1}\right), \ldots,\left(w_{\ell}, m_{\ell}\right),\left(m_{\ell}, w_{1}\right) .
$$

Then $m_{i}=\alpha\left(w_{i}\right)$ for $i=1,2, \ldots, \ell$ and $m_{i}=\beta\left(w_{i+1}\right)$ for $i=1,2, \ldots, \ell-1$ and $m_{\ell}=$ $\beta\left(w_{1}\right)$ by the construction of $G\left(\mathscr{L}_{W}, P\right)$. Let $p_{i}$ be the tally of the iteration counter $k$ when $m_{i}$ proposed to $w_{i}$, and let $r_{i}$ be the tally of $k$ when $m_{i-1}$ was rejected by $w_{i}$ in the execution of the mGS, where we use the convention that $r_{1}$ denotes the tally of $k$ when $m_{\ell}$ was rejected by $w_{1}$. Then

$$
\begin{aligned}
& p_{i}>r_{i+1} \text { for } i=1,2, \ldots, \ell-1, \\
& p_{\ell}>r_{1} \text {, } \\
& r_{i} \geq p_{i} \quad \text { for } i=1,2, \ldots, \ell \text {. }
\end{aligned}
$$

The first and the second inequalities follow from (3). The third inequality is due to the fact that $w_{i}$ rejects $m_{i-1}=\beta\left(w_{i}\right)$ only because of the engagement to or the proposal from $m_{i}=\alpha\left(w_{i}\right)$. Then we obtain

$$
p_{1}>r_{2} \geq p_{2}>r_{3} \geq p_{3}>\cdots \geq p_{\ell-1}>r_{\ell} \geq p_{\ell}>r_{1} \geq p_{1},
$$

which is a contradiction.

\footnotetext{
1 A rooted spanning tree is called an arborescence in [1].
} 
Remark 1 The rotation in a complete matching, one of the key components introduced in [5], should be defined as follows when the roles of men and women are switched. Let $f$ denote a complete matching, and let

$$
s_{f}(w):=\max _{\succ_{w}}\left\{m \in M \mid w \succ_{m} f(m)\right\} \quad \text { and } \quad \operatorname{next}_{f}(w):=f\left(s_{f}(w)\right) .
$$

Then a sequence of matched pairs $\left(w_{0}, m_{0}\right),\left(w_{1}, m_{1}\right), \ldots,\left(w_{r-1}, m_{r-1}\right)$ should be called a rotation in $f$ if

$$
w_{i+1}=\operatorname{next}_{f}\left(w_{i}\right) \quad \text { for } i=0,1, \ldots, r-1
$$

with the convention that $w_{r}=w_{0}$. When the graph $G\left(\mathscr{L}_{W}, P\right)$ contains a directed cycle $\left(w_{0}, m_{0}\right),\left(m_{0}, w_{1}\right),\left(w_{1}, m_{1}\right), \ldots,\left(w_{r-1}, m_{r-1}\right),\left(m_{r-1}, w_{0}\right)$, we will see that the reverse sequence $\left(w_{r-1}, m_{r-1}\right),\left(w_{r-2}, m_{r-2}\right), \ldots,\left(w_{1}, m_{1}\right),\left(w_{0}, m_{0}\right)$ of woman-man pairs corresponds to a rotation in the complete matching $\alpha^{-1}$. Since we lack men's preference profile $\mathscr{L}_{M}$, we replace the condition $w \succ_{m} f(m)$ in the definition of $s_{f}(w)$ by $w \in P_{M}(m) \backslash\left\{\alpha^{-1}(m)\right\}$. Note that this condition is equivalent to $m \in P_{W}(w) \backslash\{\alpha(w)\}$. By the construction of the second suitor graph $G\left(\mathscr{L}_{W}, P\right)$,

$$
m_{i}=\beta\left(w_{i+1}\right)=\max _{\succ_{w_{i+1}}}\left(P_{W}\left(w_{i+1}\right) \backslash\left\{\alpha\left(w_{i+1}\right)\right\}\right),
$$

which means that $m_{i}=s_{f}\left(w_{i}\right)$. Hence we obtain

$$
\operatorname{next}_{f}\left(w_{i+1}\right)=f\left(s_{f}\left(w_{i+1}\right)\right)=f\left(m_{i}\right)=w_{i},
$$

implying that $\left(w_{r-1}, m_{r-1}\right),\left(w_{r-2}, m_{r-2}\right), \ldots,\left(w_{1}, m_{1}\right),\left(w_{0}, m_{0}\right)$ is a rotation.

Now suppose that we are given $\mathscr{L}_{W}$ and $P$ such that the second suitor graph $G\left(\mathscr{L}_{W}, P\right)$ is a rooted spanning tree. Then $\operatorname{indeg}(m)=1$ for each node $m \in M$, that is, $\alpha(w) \neq \alpha\left(w^{\prime}\right)$ whenever $w \neq w^{\prime}$. Therefore the mapping $\hat{f}: M \cup W \rightarrow M \cup W$ defined as follows is a complete matching:

$$
\begin{array}{ll}
\hat{f}(w):=\alpha(w) & \text { for } w \in W \\
\hat{f}(m):=\alpha^{-1}(m) & \text { for } m \in M .
\end{array}
$$

Note that $\alpha^{-1}(m)=\operatorname{pred}(m)$, the predecessor of $m$ in $G\left(\mathscr{L}_{W}, P\right)$. For the complete matching $\hat{f}$ defined above, let $\mathscr{L}_{M}^{*}=\left[\succ_{m}^{*}\right]_{m \in M}$ be an arbitrary preference profile of men satisfying the following two conditions (compare with Table 1):

$$
\begin{array}{ll}
w \succ_{m}^{*} \hat{f}(m) & \text { for all } w \in P_{M}(m) \backslash\{\hat{f}(m)\}, \\
\hat{f}(m) \succ_{m}^{*} w^{\prime} & \text { for all } w^{\prime} \in W \backslash P_{M}(m) .
\end{array}
$$

Lemma 6 Suppose that the second suitor graph $G\left(\mathscr{L}_{W}, P\right)$ is a rooted spanning tree. Let $\mathscr{L}_{M}^{*}:=\left[\succ_{m}^{*}\right]_{m \in M}$ be a preference profile of men satisfying the conditions $(5)$ and (6), and let $f^{*}:=\mu\left(\mathscr{L}_{M}^{*}, \mathscr{L}_{W}\right)$. Then it holds that

$$
f^{*}(m) \succeq_{m}^{*} \hat{f}(m) \quad \text { for all } m \in M .
$$


Proof Since the complete matching $f^{*}$ is a men-optimal stable matching (see, e.g., Theorem 1.2.2 in [5]), it suffices to show that $\hat{f}$ is a stable matching with respect to $\mathscr{L}_{M}^{*}$ and $\mathscr{L}_{W}$. Let $\{m, w\}$ be an arbitrary unmatched pair in $\hat{f}$ and suppose that $w \succ_{m}^{*} \hat{f}(m)$. Then by (5) we see that $w \in P_{M}(m)$, which implies that $m \in P_{W}(w)$. Therefore $\hat{f}(w)=\alpha(w)=\max _{\succ_{w}} P_{W}(w) \succeq_{w} m$. This means that there are no blocking pairs for $\hat{f}$, hence $\hat{f}$ is stable.

Theorem 1 Let $\mathscr{L}_{W}$ be a preference profile of women, and $P \subseteq M \times W$ be a set of proposals. There is a preference profile of men $\mathscr{L}_{M}^{*}$ such that $\Pi\left(\mathscr{L}_{M}^{*}, \mathscr{L}_{W}\right)=P$ if and only if the second suitor graph $G\left(\mathscr{L}_{W}, P\right)$ is a rooted spanning tree. In this case, $\Pi\left(\mathscr{L}_{M}^{*}, \mathscr{L}_{W}\right)=P$ holds if and only if $\mathscr{L}_{M}^{*}$ satisfies (5) and (6).

Proof We have seen that if there is $\mathscr{L}_{M}^{*}$ such that $\Pi\left(\mathscr{L}_{M}^{*}, \mathscr{L}_{W}\right)=P$, then $G\left(\mathscr{L}_{W}, P\right)$ is a rooted spanning tree, and also that $\mathscr{L}_{M}^{*}$ satisfies (5) and (6). We then suppose that $G\left(\mathscr{L}_{W}, P\right)$ is a rooted spanning tree, and will show that $\mu\left(\mathscr{L}_{M}^{*}, \mathscr{L}_{W}\right)=\hat{f}$ for any $\mathscr{L}_{M}^{*}$ satisfying (5) and (6). This implies the desired result that $\Pi\left(\mathscr{L}_{M}^{*}, \mathscr{L}_{W}\right)=P$ by the construction of $\mathscr{L}_{M}^{*}$.

Denoting $\mu\left(\mathscr{L}_{M}^{*}, \mathscr{L}_{W}\right)$ by $f^{*}$ for the sake of simplicity, we will prove $f^{*}(u)=$ $\hat{f}(u)$ by the induction over the height $h(u)$ of node $u$ on $G\left(\mathscr{L}_{W}, P\right)$. Note that $h(u)$ is odd when $u \in W$, and even when $u \in M$.

- For $w \in W$ with $h(w)=1$ :

Let $m^{\prime}:=f^{*}(w)$. Then by Lemma 6 we have $w=f^{*}\left(m^{\prime}\right) \succeq_{m^{\prime}}^{*} \hat{f}\left(m^{\prime}\right)$. This means that $w \in P_{M}\left(m^{\prime}\right)$ by (6), hence $m^{\prime} \in P_{W}(w)$. Since $h(w)=1$, i.e., an arc comes in from the root $r, P_{W}(w)$ is a singleton set of $\hat{f}(w)$ by Definition 7 . Therefore we have $m^{\prime}=\hat{f}(w)$.

- For $m \in M$ :

Suppose that $f^{*}(w)=\hat{f}(w)$ for all $w \in W$ with $h(w)=h(m)-1$ as the induction hypothesis. Let $w:=\hat{f}(m)$. Then $h(w)=h(m)-1$ and we have $f^{*}(\hat{f}(m))=f^{*}(w)=$ $\hat{f}(w)=\hat{f}(\hat{f}(m))=m$. Since $f^{*}$ is a complete matching, we obtain $\hat{f}(m)=\left(f^{*}\right.$ o $\left.f^{*}\right)(\hat{f}(m))=f^{*}\left(f^{*}(\hat{f}(m))\right)=f^{*}(m)$.

- For $w \in W$ with $h(w) \geq 3$ :

Let $m:=\operatorname{pred}(w)$ in $G\left(\mathscr{L}_{W}, P\right)$. Then $m \neq \hat{f}(w)$, since otherwise the graph $G\left(\mathscr{L}_{W}, P\right)$ would contain a directed cycle $(w, m),(m, w)$, contradicting the assumption. Since $\hat{f}$ is a complete matching, $\hat{f}(m) \neq w$. Since $m \in P_{W}(w)$, we have

$$
w \in P_{M}(m) \backslash\{\hat{f}(m)\} .
$$

Therefore by (5) we obtain

$$
w \succ_{m}^{*} \hat{f}(m) .
$$

By the induction hypothesis $f^{*}(m)=\hat{f}(m)$, which we have seen is different from $w$. This means that $m$ was rejected by $w$ in the execution of the mGS. Therefore $f^{*}(w) \succ_{w} m$. Namely, letting $M_{\succ_{w} m}:=\left\{m^{\prime} \in M \mid m^{\prime} \succ_{w} m\right\}$, we have $f^{*}(w) \in M_{\succ_{w} m}$.

Now let $m^{*}:=f^{*}(w)$ and we will show that $m^{*} \neq \hat{f}(w)$ leads to a contradiction. Since $M_{\succ_{w} m} \cap P_{W}(w)=\{\hat{f}(w)\}$ and $m^{*} \in M_{\succ_{w} m}$, we have that $m^{*} \notin P_{W}(w)$, which 
implies $w \notin P_{M}\left(m^{*}\right)$. Then by (6) we have

$$
\hat{f}\left(m^{*}\right) \succ_{m^{*}}^{*} w=\left(f^{*} \circ f^{*}\right)(w)=f^{*}\left(f^{*}(w)\right)=f^{*}\left(m^{*}\right) .
$$

This contradicts Lemma 6 . Thus we have the equality that $f^{*}(w)=\hat{f}(w)$.

Example 3 Let $M:=\{1,2,3,4,5,6,7\}$ and $W:=\{a, b, c, d, e, f, g\}$, and let $\mathscr{L}_{W}$ be given in the left table of Table 3, where the elements of $P_{W}(w)$ are underlined and $\alpha(w)$ is in bold face for each $w \in W$. An example of men's preference profile $\mathscr{L}_{M}^{*}$ satisfying (2), (3) and (4) is given in the right table. The second suitor graph $G\left(\mathscr{L}_{W}, P\right)$ is shown in Figure 1.

Table $3 \mathscr{L}_{W}$ and $\mathscr{L}_{M}^{*}$

\begin{tabular}{c|ccccccc}
$w$ & \multicolumn{7}{|c}{$\mathscr{L}_{W}$} \\
\hline$a$ & $\underline{\mathbf{1}}$ & 2 & $\underline{3}$ & 4 & $\underline{5}$ & $\frac{7}{7}$ & 6 \\
$b$ & 6 & $\underline{\mathbf{2}}$ & 1 & $\underline{3}$ & $\underline{4}$ & 5 & 4 \\
$c$ & $\underline{\mathbf{3}}$ & 1 & 6 & 5 & 2 & 7 & 4 \\
$d$ & $\mathbf{7}$ & $\underline{\mathbf{4}}$ & $\underline{3}$ & 6 & $\underline{2}$ & $\underline{5}$ & $\underline{1}$ \\
$e$ & 2 & 7 & $\underline{\mathbf{5}}$ & 1 & $\underline{6}$ & $\underline{3}$ & 4 \\
$f$ & 4 & 1 & 3 & $\underline{\mathbf{6}}$ & $\frac{7}{2}$ & 5 \\
$g$ & 1 & $\underline{\mathbf{7}}$ & $\underline{6}$ & $\underline{4}$ & $\underline{5}$ & $\underline{3}$ & $\underline{2}$
\end{tabular}

\begin{tabular}{c|ccccccc}
$m$ & \multicolumn{1}{|c}{$\mathscr{L}_{M}^{*}$} \\
\hline 1 & $\underline{d}$ & $\underline{\mathbf{a}}$ & $b$ & $c$ & $e$ & $f$ & $g$ \\
2 & $\underline{d}$ & $\underline{g}$ & $\underline{\mathbf{b}}$ & $a$ & $c$ & $e$ & $f$ \\
3 & $\underline{a}$ & $\underline{b}$ & $\underline{d}$ & $\underline{e}$ & $\underline{g}$ & $\underline{\mathbf{c}}$ & $f$ \\
4 & $\underline{b}$ & $\underline{g}$ & $\underline{\mathbf{d}}$ & $a$ & $c$ & $e$ & $f$ \\
5 & $\underline{a}$ & $\underline{d}$ & $\underline{g}$ & $\underline{\mathbf{e}}$ & $b$ & $c$ & $f$ \\
6 & $\underline{e}$ & $\underline{g}$ & $\underline{\mathbf{f}}$ & $a$ & $b$ & $c$ & $d$ \\
7 & $\underline{a}$ & $\underline{\mathbf{g}}$ & $b$ & $c$ & $d$ & $e$ & $f$
\end{tabular}

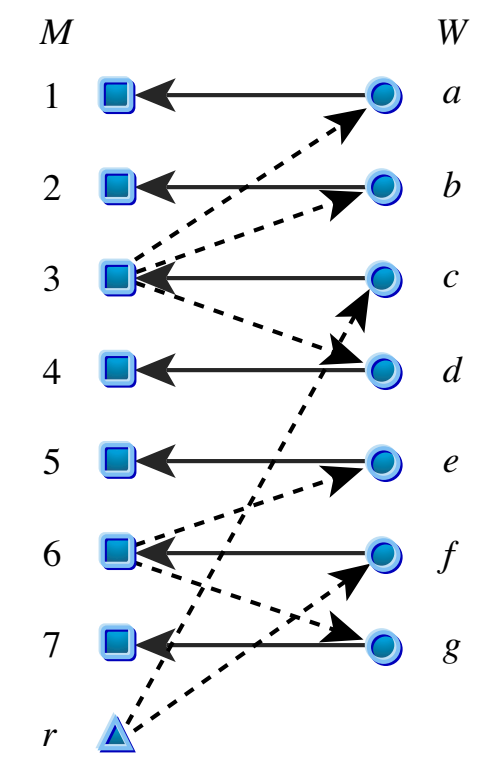

Fig. 1 Second suitor graph for $\mathscr{L}_{W}$ and $P$ 


\section{Existence of preference profiles of men and women}

We have considered the existence problem of a preference profile of men $\mathscr{L}_{M}$ when a preference profile of women $\mathscr{L}_{W}$ and a set of proposals $P$ are given in the preceding sections. A natural question to pose would be whether there is a pair of $\mathscr{L}_{M}$ and $\mathscr{L}_{W}$ that makes the mGS produce $P$ and/or a complete matching $f$. We will first show in the following subsection that the problem is solvable in polynomial time when $f$ as well as $P$ is given. Then we will show it is still polynomially solvable when $P$ alone is given.

6.1 Case where $f$ and $P$ are given

Corollary 1 Let $\mathscr{L}_{M}$ and $\mathscr{L}_{W}$ be preference profiles of men and women, respectively, and let $\mu:=\mu\left(\mathscr{L}_{M}, \mathscr{L}_{W}\right)$ and $\Pi:=\Pi\left(\mathscr{L}_{M}, \mathscr{L}_{W}\right)$ be the output of the $m G S$. Let $\mathscr{L}_{M}^{*}=$ $\left[\succ_{m}^{*}\right]_{m \in M}$ be an arbitrary preference profile of men such that

$$
w \succ_{m}^{*} \mu(m) \succ_{m}^{*} w^{\prime} \quad \text { for all } w \in \Pi_{M}(m) \backslash\{\mu(m)\} \text { and } w^{\prime} \in W \backslash \Pi_{M}(m)
$$

holds for all $m \in M$. Then $\mu\left(\mathscr{L}_{M}, \mathscr{L}_{W}\right)=\mu\left(\mathscr{L}_{M}^{*}, \mathscr{L}_{W}\right)$ and $\Pi\left(\mathscr{L}_{M}, \mathscr{L}_{W}\right)=\Pi\left(\mathscr{L}_{M}^{*}, \mathscr{L}_{W}\right)$.

Proof The second suitor graph $G\left(\mathscr{L}_{W}, \Pi\left(\mathscr{L}_{M}, \mathscr{L}_{W}\right)\right)$ is a rooted spanning tree by Theorem 1 . Then, by the same theorem, the mGS produces $\Pi\left(\mathscr{L}_{M}, \mathscr{L}_{W}\right)$ as well as $\mu\left(\mathscr{L}_{M}, \mathscr{L}_{W}\right)$ for any preference profile of men $\mathscr{L}_{M}^{*}$ satisfying (7).

Now suppose that we are given a complete matching $f$ and a set of proposals $P$ such that

$$
(m, f(m)) \in P \quad \text { for all } m \in M .
$$

If there is a pair of preference profiles $\mathscr{L}_{M}$ and $\mathscr{L}_{W}$ that makes the mGS produce $f$ and $P, \mathscr{L}_{M}$ must satisfy

$$
w \succ_{m} f(m) \succ_{m} w^{\prime} \quad \text { for all } w \in P_{M}(m) \backslash\{f(m)\} \text { and } w^{\prime} \in W \backslash P_{M}(m) .
$$

Now let $\mathscr{L}_{M}^{*}$ be an arbitrary preference profile of men satisfying (9), and consider the existence problem of Kobayashi and Matsui [7] or the first problem in [8]. If their polynomial time algorithm provides a preference profile of women $\mathscr{L}_{W}^{*}$ that together with $\mathscr{L}_{M}^{*}$ makes the mGS produce $f$, we are done. If not, by Corollary 1 we conclude that there are no preference profiles of women for any preference profile of men satisfying (9). Namely, no pairs of preference profiles of men and women make the mGS produce the prescribed complete matching $f$ or the prescribed set of proposals $P$. Thus we obtain the following theorem.

Theorem 2 Given a set of proposals $P$ and a complete matching $f$ satisfying (8), the existence problem of preference profiles $\mathscr{L}_{M}$ and $\mathscr{L}_{W}$ such that $P=\Pi\left(\mathscr{L}_{M}, \mathscr{L}_{W}\right)$ and $f=\mu\left(\mathscr{L}_{M}, \mathscr{L}_{W}\right)$ is solvable in polynomial time. 
6.2 Case where $P$ alone is given

Definition 11 For a given set of proposals $P$ let $H(P)=((M \cup\{r\}) \cup W, E)$ be the undirected graph with the node set $(M \cup\{r\}) \cup W$ and the edge set $E$ defined by

$$
E:=\{\{m, w\} \mid(m, w) \in P\} \cup\left\{\{r, w\}|w \in W,| P_{W}(w) \mid=1\right\} .
$$

For each node $v \in(M \cup\{r\}) \cup W$ let $\delta(v)$ denote the set of edges incident to $v$ and let $\operatorname{deg}(v):=|\delta(v)|$ and call it the degree of $v$.

Lemma 7 If $H(P)$ has no spanning tree such that

$$
\operatorname{deg}(w)=2 \quad \text { for all } w \in W,
$$

there are no pairs of $\mathscr{L}_{M}$ and $\mathscr{L}_{W}$ such that $\Pi\left(\mathscr{L}_{M}, \mathscr{L}_{W}\right)=P$.

Proof Suppose that there are $\mathscr{L}_{M}$ and $\mathscr{L}_{W}$ such that $\Pi\left(\mathscr{L}_{M}, \mathscr{L}_{W}\right)=P$. Then the undirected version of the second suitor graph $G\left(\mathscr{L}_{W}, P\right)$ is a spanning tree of $H(P)$ satisfying the degree constraint (10) from Lemma 2 and the proof of Theorem 1.

Now suppose that the graph $H(P)$ has a spanning tree $T$ satisfying the degree constraint (10). Give orientations to the edges of $T$ so that it becomes a rooted spanning tree with $r$ as the root. We denote this rooted spanning tree by $T^{*}$. Then $\operatorname{indeg}(w)=\operatorname{outdeg}(w)=1$ for each $w \in W$, hence its predecessor $\operatorname{pred}(w)$ and successor $\operatorname{succ}(w)$ in $T^{*}$ are uniquely determined. Now let $\succ_{w}^{*}$ denote an arbitrary preference list of $w \in W$ satisfying

$$
\operatorname{succ}(w) \succ_{w}^{*} \operatorname{pred}(w) \succ_{w}^{*} m \quad \text { for all } m \in P_{W}(w) \backslash\{\operatorname{succ}(w), \operatorname{pred}(w)\}
$$

and collect them to make a preference profile of women, which we will denote by $\mathscr{L}_{W}^{*}$.

Lemma 8 If $H(P)$ has a spanning tree satisfying the degree constraint (10), then there is a pair of $\mathscr{L}_{M}$ and $\mathscr{L}_{W}$ such that $\Pi\left(\mathscr{L}_{M}, \mathscr{L}_{W}\right)=P$.

Proof As discussed above, the second suitor graph $G\left(\mathscr{L}_{W}^{*}, P\right)$ turns out to be a rooted spanning tree for the preference profile of women $\mathscr{L}_{W}^{*}$ being a collection of preference lists $\succ_{w}^{*}$ satisfying (11) and the given set of proposals $P$. Then by Theorem 1 there is a preference profile of men $\mathscr{L}_{M}$ such that $\Pi\left(\mathscr{L}_{M}, \mathscr{L}_{W}^{*}\right)=P$.

The problem of fining a degree-constrained spanning tree $T$ in the graph $H(P)$ reduces to a matroid intersection problem of two matroids defined on $E$. One matroid is the graphic matroid, whose independent sets are cycle-free sets of edges; the other is the partition matroid, where a subset $I$ of $E$ is independent if and only if

$$
|I \cap \delta(w)| \leq 2 \text { for all } w \in W .
$$

Clearly, a common basis, if any, is a spanning tree of $H(P)$ satisfying the degree constraint (10), and vice versa. 
Theorem 3 Given a set of proposals $P$, the existence problem of a pair of $\mathscr{L}_{M}$ and $\mathscr{L}_{W}$ such that $\Pi\left(\mathscr{L}_{M}, \mathscr{L}_{W}\right)=P$ is solvable in polynomial time.

Proof Since the matroid intersection problem of a graphic matroid and a partition matroid is solvable in polynomial time, we obtain the theorem.

See [2], [3] and [9] for algorithms for the matroid intersection problem and their computational complexity.

\section{Conclusion}

According to [8], one of the open questions as to the strategic manipulability in the stable matching model is described as "given a pair of preference profiles $\mathscr{L}_{M}$ and $\mathscr{L}_{W}$, is there a preference profile of women $\mathscr{K}_{W}$ such that $\mu\left(\mathscr{L}_{M}, \mathscr{K}_{W}\right)$ is a stable matching with respect to $\mathscr{L}_{M}$ and $\mathscr{L}_{W}$ and is different from the pessimal stable matching $\mu\left(\mathscr{L}_{M}, \mathscr{L}_{W}\right)$ ?" Switching the roles of men and women yields a question "is there a preference profile of men $\mathscr{K}_{M}$ such that $\mu\left(\mathscr{K}_{M}, \mathscr{L}_{W}\right)$ is a stable matching with respect to $\mathscr{L}_{M}$ and $\mathscr{L}_{W}$ and is different from $\mu\left(\mathscr{L}_{M}, \mathscr{L}_{W}\right)$ ?" It would be satisfying if the results in this paper make a dent in these problems.

Acknowledgements We are very grateful to Tomomi Matsui, Chuo University, and Akihisa Tamura, Keio University for their stimulating discussion, and also anonymous referees for their meticulous reading of an earlier version of this paper. The second author is partly supported by Grant-in-Aid for Scientific Research (C) 22510136 .

\section{References}

1. Berge, C. : Graphs and Hypergraphs, North-Holland, Amsterdam (1973)

2. Fujishige, S : A primal approach to the independent assignment problem, Journal of the Operations Research Society of Japan, 20, 1-15 (1977)

3. Gabow, H.N., Tarjan, R.E. : Efficient algorithms for a family of matroid intersection problems, Journal of Algorithms, 5, 80-131 (1984)

4. Gale, D., Shapley, L.S. : College admissions and stability of marriage, American Mathematical Monthly, 69, 9-15 (1962)

5. Gusfield, D., Irving, R.W. : The Stable Marriage Problem: Structure and Algorithms, MIT Press, Cambridge (1989)

6. Knuth, D.E. : Mariages stables et leurs relations avec d'autres problèmes combinatoires, Les Presses de l'Universitè de Montrèal (1976), English translation by M. Goldstein: Stable marriage and its relation to other combinatorial problems, CRM Proceedings and Lecture Notes Vol.10, American Mathematical Society, Providence (1997)

7. Kobayashi, H., Matsui, T. : Successful manipulation in stable marriage model with complete preference lists, IEICE Trans. Inf. Syst. E92-D, 116-119 (2009)

8. Kobayashi, H., Matsui, T. : Cheating strategies for the Gale-Shapley algorithm with complete preference lists, Algorithmica, 58, 151-169 (2010)

9. Lawler, E.L. : Combinatorial Optimization : Network and Matroids, Holt, Rinehart and Winston, New York (1976)

10. Teo, C.-T., Sethuraman, J., Tan, W.-P.: Gale-Shapley stable marriage problem revisited: strategic issues and applications, Management Sciences 47, 1252-1267 (2001) 